Marquette University

e-Publications@Marquette

Exercise Science Faculty Research and Publications

Exercise Science, Department of

7-1-2009

Sex Differences and Mechanisms of Task-Specific Muscle Fatigue

Sandra K. Hunter

Marquette University, sandra.hunter@marquette.edu

Accepted version. Exercise and Sport Sciences Review, Vol. 37, No. 3 (2009): 113-122. DOI. (C) 2009 Lippincott Williams and Wilkins, Inc. Used with permission. 


\title{
Sex Differences and Mechanisms of Task-Specific Muscle
}

\section{Fatigue}

\author{
Sandra K. Hunter, Ph.D., FACSM \\ Exercise Science Program, Department of Physical Therapy, Marquette University
}

\begin{abstract}
Women can be less fatigable than men due to sex-related differences within the neuromuscular system that impact physiological adjustments during a fatiguing task. The involved mechanism(s) for the sex difference, however, is task specific. This review explores the novel hypothesis that variation of the task will alter the magnitude of the sex-difference in muscle fatigue and the contribution of involved mechanisms.
\end{abstract}

\section{Keywords}

Gender; time to task failure; stress; aging; isometric contractions; dynamic contractions

\section{INTRODUCTION}

Muscle fatigue is classically defined as an exercise-induced decline in maximal voluntary muscle force or power (5). It develops in men and women soon after the onset of sustained physical activity and is often quantified as a reduction in maximal voluntary contraction (MVC) force after or during maximal and submaximal exercise (5). Muscle fatigue can occur despite continued and successful performance of a submaximal task. If the submaximal task is maintained, however, task failure will eventually occur (16). Failure of a task can occur because the force or power requirements of the submaximal task are greater than the maximal strength or power able to be exerted by the principal muscles $(15,16)$. Task failure may also involve other rate-limiting physiological adjustments that occur during a fatiguing contraction (5). Insight into what causes task failure can be achieved by comparing physiological adjustments with variation in the task, sex of the individual, or the age of the person performing the task. This functionally relevant approach has shed light on the reasons men and women differ in performance of a fatiguing contraction.

Women exhibit different fatigue characteristics than men. For example, when men and women perform a submaximal fatiguing contraction at the same relative intensity, women are often able to sustain the contraction for a longer duration than men before task failure occurs $(3,11,17,19)$. Similarly, women experience less of a reduction in maximal force during sustained or intermittent maximal contractions $(12,28)$. Potential mechanisms for the sex difference in muscle fatigue were reviewed by Hicks and colleagues in Exercise Sport

\footnotetext{
Author Contact Information: Sandra K Hunter, Ph.D., FACSM, Exercise Science Program, Department of Physical Therapy, PO Box 1881, Milwaukee, WI, 53201, Sandra.Hunter@marquette.edu, Tel: 414288 6673, Fax: 4142886079.

Publisher's Disclaimer: This is a PDF file of an unedited manuscript that has been accepted for publication. As a service to our customers we are providing this early version of the manuscript. The manuscript will undergo copyediting, typesetting, and review of the resulting proof before it is published in its final citable form. Please note that during the production process errors may be discovered which could affect the content, and all legal disclaimers that apply to the journal pertain.
} 
Sciences Reviews in 2001 (11) and was based on a small but growing number of studies. Renewed interest in this topic has highlighted the concept that the magnitude of the sexdifference is specific to the task performed and that these differences can change across the lifespan.

The sex-difference in muscle fatigue and its dependence on task are illustrated collectively in Figures 1 and 2. Figure 1 shows the mean relative difference in performance (muscle fatigue or time to task failure) between young men and women for an isometric sustained task (Fig. 1A), isometric intermittent task (Fig. 1B), and dynamic task (Fig. 1C), at various contraction intensities in 42 studies. In all three panels, a data point above the line indicates that the mean time to task failure was greater or the reduction in maximal force was less for the young women compared with the young men in one study at the plotted intensity of contraction (x-axis). Most studies indicate a sex difference that corresponds to young women being less fatigable than men when performing isometric contractions that are sustained (Fig. 1A) and intermittent (Fig. 1B). In contrast, there was no systematic sex difference during shortening contractions, except when the task involved the elbow flexor muscles at intermediate contraction intensities (Fig. 1C).

Similarly, Figure 2 illustrates the influence of age on the sex difference in performance during a fatiguing contraction. Figure 2 is a summary of 40 studies showing the mean difference in muscle fatigue between men and women for an isometric and dynamic task as a function of the mean age of the subject group assessed within a study. The reduced sex difference in muscle fatigability among the older age groups is marked. Taken together, Figures 1 and 2 provide evidence that the magnitude of the sex difference in muscle fatigue depends on the task details, including the type of contraction performed, intensity of the contraction, and age of the person performing the task.

The question that arises from these observations is "why does the magnitude of the sexdifference in performance during a fatiguing contraction vary with the task performed?" The answer involves understanding two main concepts: 1) the involved mechanisms for task failure are specific to the details of the task during a fatiguing contraction, and 2) sex-related differences within the neuromuscular system will impact physiological adjustments during a fatiguing task in men and women differently. Task specificity of muscle fatigue will be addressed briefly, followed by a more comprehensive discussion of the recent findings that support the novel hypothesis that the role of a contributing mechanism to the sex difference in performance during a fatiguing contraction changes with variation in the task.

\section{MUSCLE FATIGUE IS TASK SPECIFIC}

The decline in force during a fatiguing task may be due to impairments at a number of sites within the neuromuscular system, ranging from an inadequate activation of the motor cortex to impairment of the contractile proteins within skeletal muscle. Multiple mechanisms may contribute to force impairment during a fatiguing contraction with the dominant mechanism specific to the process that is stressed the most (9). The mechanisms responsible for muscle fatigue and ultimately failure of a task, however, are specific to the details of the task performed (5). This is because varying the task requirements will stress different sites within the neuromuscular system and therefore will influence the magnitude of muscle fatigue or time to failure of a task. Consequently, there is not one single or global cause of muscle fatigue because the potential mechanisms vary according to the requirements of the task. Insight on possible mechanisms can be gained by comparing physiological adjustments during a fatiguing contraction as the task is varied $(5,16)$. Some variables that influence the involved mechanisms include the type and intensity of contraction, the muscle groups involved, the limb support provided and the physical environment in which the task is 
performed $(5,16)$. For some of these task variables, the sex of the individual will influence the magnitude of muscle fatigue and the strength of the contributing mechanisms.

Sex-related differences within the neuromuscular system will impact physiological adjustments that occur during a fatiguing task performed by men and women. Furthermore, the dominant mechanisms for the sex difference will change when that task is varied due to a shift in the stress on the different processes within the neuromuscular system. Consequently, there is no single mechanism that can explain the sex difference in performance of a fatiguing contraction. Figure 3 illustrates some potential mechanisms that can contribute to the sex difference in muscle fatigue and failure of a task when women are less fatigable: the strength of these potential contributing mechanisms to the sex difference in performance of fatiguing contraction, however, will vary with the involved task.

Potential mechanisms and physiological adjustments that may occur during a fatiguing contraction are shown that can result in women being less fatigable than men for tasks that are exerted at the same relative intensity by both sexes. A negative sign in Figure 3 indicates that the physiological variable or adjustment during the contraction is less in women than men and, conversely, a positive sign denotes a greater adjustment in women than men. Some of the shown mechanisms are highlighted in the subsequent discussion in this review. The diagram in Figure 3 however, does not indicate the strength of a mechanism for any one specific task. Some mechanisms (for example, fiber types) could be responsible for women being more fatigue resistant than men during isometric contractions. In contrast, a greater proportional area of Type I fibers in the muscles of women may be responsible for the lack of a sex difference during dynamic contractions when the requirements are to maintain a given velocity. Likewise, a sex difference in voluntary activation is probably only relevant to certain muscle groups such as knee extensor and ankle dorsiflexor muscles, but not relevant to the elbow flexor muscles. Furthermore, when the task and environmental conditions change so that women perceive they are more stressed than under control conditions, the sex difference in time to failure of a task can be diminished. These and other examples are highlighted in this review. Thus, rather than address the potential individual mechanisms in Figure 3, the second part of this review will examine how variation of selected tasks will alter the influence of mechanisms that contribute to the sex difference in performance of a fatiguing contraction.

\section{CONTRACTION INTENSITY}

The intensity at which an isometric contraction is performed by men and women will influence the time to failure of a task for the fatiguing contraction and also the magnitude of the sex difference in performance. The average magnitude of the sex difference across all intensities for sustained isometric contractions is $\sim 23 \%$ (relative to the fatigue index or time to failure of the women as graphed in Figure 1A), indicating that women are less fatigable than men for this task. The magnitude of this sex difference, however, depends on contraction intensity so that the sex difference in muscle fatigue is larger at lower relative contraction intensities. For example, the time to task failure for a contraction sustained at $20 \%$ of maximum strength with the elbow flexor muscles was longer for young women than young men (mean $\pm \mathrm{SD}$ : $17.0 \pm 8.7 \mathrm{~min}$ vs $10.6 \pm 2.0 \mathrm{~min}$, respectively) for men $(88 \pm 22$ $\mathrm{N} \bullet \mathrm{m})$ who were stronger than women $(45 \pm 7 \mathrm{~N} \bullet \mathrm{m})(35)$. The time to failure, however, was similar for the same young women and men when a contraction was sustained at $80 \%$ of maximum strength until task failure ( $24.3 \pm 6.6 \mathrm{~s}$ vs $25.0 \pm 6.5 \mathrm{~s})$ (35). The negative relation between contraction intensity and the magnitude of the sex difference in time to task failure or muscle fatigue is illustrated in Figure 1A as a negative linear slope $\left(\mathrm{r}^{2}=0.42\right)$ among all the muscle groups reviewed for sustained contractions. 
The reason for the negative relation between contraction intensity of sustained contractions and the sex difference in muscle fatigue is related to the absolute strength of men and women when exerting the same relative contraction intensity. This was illustrated in several studies that compared the time to task failure of the strength-matched men and women during low-force contractions. Ten young men and 10 young women were matched in pairs within $5 \%$ of absolute strength with the elbow flexor muscles $(64.5 \pm 8.7 \mathrm{~N} \bullet \mathrm{m}$ vs $64.5 \pm 8.3$ $\mathrm{N} \bullet \mathrm{m}$, respectively) (14). The time to task failure of a fatiguing contraction sustained at $20 \%$ of maximal strength was similar for the men $(13.7 \pm 5.1 \mathrm{~min})$ and women $(14.4 \pm 6.9 \mathrm{~min})$ (14) (Fig. 4, Study 2). The rate of increase in mean arterial pressure (pressor response) during the fatiguing contraction was also similar for the men and women. The similar pressor response indicated that the attempt by the central nervous system to maintain perfusion in the target muscle was also similar for the men and women. In contrast, when men were stronger than women for the elbow flexor muscles, the time to task failure for a $20 \%$ sustained contraction was longer for the women than the men (Fig. 4, Study 1), and the pressor response increased at a lesser rate for the women than the men $(13,17,35)$. Similar to the elbow flexor muscles, the sex difference in time to task failure (men, $8.6 \pm 2.4 \mathrm{~min}$ vs women, $11.4 \pm 2.8 \mathrm{~min}$ ) when men were stronger than women for the finger flexor muscles (handgrip) disappeared when men and women were matched for strength (men, $8.4 \pm 1.6$ min vs women, $8.6 \pm 2.3 \mathrm{~min})(19)$.

So why does strength determine the fatigability of muscle in men and women especially during low-force sustained isometric contractions? One possibility is that large absolute contractions exerted by the men involve greater intramuscular pressures and occlusion of blood flow during static contractions than women, a more rapid accumulation of metabolites, impairment of oxygen delivery to the muscle, and a more rapid rate of muscle fatigue (11) (Fig. 3). Associations between the absolute forces exerted during a relative task and the time to failure in young adults, and the pressor response (change in mean arterial pressure) $(13,17,35)$ are consistent with the interpretation that blood flow and delivery of oxygen to the working muscle may be more occluded for men than women at lower forces during a sustained contraction. During high-force sustained isometric contractions, blood flow is more likely to be occluded for both men and women so that the sex difference in perfusion and time to failure of the task is reduced.

The importance of blood flow and oxygen delivery in determining the sex difference in time to task failure was illustrated in a study of the quadriceps when blood flow was artificially occluded during a low-force sustained contraction (3). Women had a longer time to task failure than men for a contraction sustained at $25 \%$ of maximal strength under conditions when the flow to the muscle was not artificially occluded $(215 \pm 21 \mathrm{~s}$ vs $169 \pm 21 \mathrm{~s}$ respectively) but a similar time to task failure when the muscle was made ischemic with a cuff ( $180 \pm 20 \mathrm{~s}$ vs $165 \pm 20 \mathrm{~s}$, respectively). More recent studies have directly measured limb flow of men and women during a contraction and determined hyperemia after isometric contractions $(19,31)$. Active hyperemia was larger for stronger men compared with women after brief contractions at the same relative intensity (19), which suggests that women had greater perfusion during the contraction than men. These studies, however, have not indicated a clear difference in perfusion between men and women during the sustained fatiguing contraction at least for forearm muscles $(19,31)$. Nevertheless, the strength of the men and women is a contributing factor to the magnitude of the sex difference in muscle fatigue for low-force sustained contractions.

\section{SUSTAINED AND INTERMITTENT ISOMETRIC TASKS}

Comparison of sustained and intermittent isometric tasks indicates that mechanisms other than absolute strength contribute to the sex difference in the rate of muscle fatigue during an 
intermittent task. The collective studies graphed in Figure 1 show a mean 33\% sex difference (relative to women) for the intermittent isometric voluntary contractions (electrically-evoked contractions not included) compared with sustained isometric contractions ( $23 \%$ relative to the women). Despite the lesser number of studies involving intermittent contractions, comparison of the relative differences among studies in Figure 1A and $1 \mathrm{~B}$ suggest that the sex difference is greater during the voluntary intermittent contractions than sustained contractions $(P=0.04)$.

A fundamental difference between the sustained and intermittent fatiguing contraction is that blood flow is not a limiting factor during the intermittent task when the ratio of contraction time to rest is not large (18). In contrast to the sustained isometric contractions, the sex difference in muscle fatigue for an intermittent isometric task does not depend on the absolute muscle strength exerted by the young adult. This was demonstrated in a study where young women were matched for strength with young men (within 5\%) and sustained an intermittent isometric contraction ( $5 \mathrm{~s}$ on and $5 \mathrm{~s}$ off) at $50 \%$ of maximal strength until task failure with the elbow flexor muscles (15). The young women $(23.5 \pm 18.9 \mathrm{~min})$ sustained the intermittent task for a longer duration than the men (8.6 $\pm 3.2 \mathrm{~min})$ (Fig. 4, Study 3 ) and the rate of decline in MVC force during the contraction (measured every minute) decreased at a slower rate for the women than the men. Similar findings were also reported for a small hand muscle (adductor pollicis) when men and women were matched for strength (136 $\pm 4 \mathrm{~N}$ vs $132 \pm 5 \mathrm{~N}$, respectively) (8). The women were able to maintain the intermittent contraction ( $5 \mathrm{~s}$ on, $5 \mathrm{~s}$ off) at $50 \% \mathrm{MVC}$ for a longer duration than the men (14.7 \pm 1.6 vs $7.9 \pm 0.7 \mathrm{~min}$, respectively) (8). Thus, the mechanisms responsible for the sex difference in fatigue and time to task failure for an intermittent task appear to differ from those responsible for the sex differences during sustained isometric contractions. Other mechanisms besides target force/muscle mass (illustrated in Fig. 3) must be responsible for the sex difference in performance of an intermittent isometric contraction.

The importance of blood flow and oxygen delivery for the sex difference in muscle fatigue during an intermittent task was illustrated in a study by Russ and Kent-Braun (28) with lower leg muscles. Men and women performed 4 min. of maximal intermittent isometric contractions ( $5 \mathrm{~s}$ on: $5 \mathrm{~s}$ off) with the dorsiflexor muscles on two separate days. Women exhibited less muscle fatigue (decrease in maximal voluntary contraction (MVC) force) than men when flow was not artificially occluded with a cuff around the limb (women, $79 \pm 4 \%$ of initial MVC; men, $66 \pm 3 \%$ of initial MVC) but similar muscle fatigue compared with men when the blood flow was occluded to the lower leg (women, $22 \pm 4 \%$ of initial MVC; men, $21 \pm 4 \%$ of initial MVC) (28). The greater fatigue for men during the non-occluded condition involved greater fatigue within the central nervous system (larger reductions in voluntary drive to the muscle) than the women. The sex difference in reduced voluntary drive was attributed to inhibitory sensory feedback to the motor neuron pool probably because of a difference in the accumulation of muscle metabolites between men and women (see Fig. 3). Thus, a sex difference in muscle metabolism by-products $(22,29)$ may have contributed to the sex difference in performance during the non-occluded condition (28). Accordingly, men have greater reliance on glycolytic pathways than women during isometric tasks with the dorsiflexor muscles $(22,29)$ (see Fig. 3). This study, however, showed no sex difference in the change in contractile properties for the tibialis anterior that would be expected to change in parallel with changes in metabolism. Nor has a direct link between a sex difference in muscle fatigue and a sex difference in muscle metabolism been established in a study with the dorsiflexor muscles $(22,29)$. The contribution of a sex difference in metabolic pathways that ensure adequate ATP to any sex difference in muscle fatigue may be more obvious for other muscle groups, especially those that show a sex difference in fiber composition (Fig. 3). 
Contractile properties measured from contractions evoked by electrical stimulation and transcranial magnetic stimulation are consistent with a sex difference in the proportional area of fiber types in the elbow flexors (12) and knee extensors (33). Women have slower rates of relaxation of the evoked contraction before fatigue for these muscles $(12,33)$ and this is consistent with larger proportional area of fast-twitch fibers in the men than the women (for example: (27)). Such differences predispose women to less muscle fatigue during an intermittent fatiguing contraction (Fig. 3). For example, endurance capacity of animal muscle assessed using electrically evoked contractions depends on fiber type composition. Consistent with this hypothesis, young women demonstrated less muscle fatigue ( $30 \pm 10 \%$ reduction) than young men ( $38 \pm 11 \%$ reduction) at the end of $2 \mathrm{~min}$. of electrically evoked intermittent contractions ( $1 \mathrm{~s}$ on at $30 \mathrm{~Hz} ; 1 \mathrm{~s}$ off) of the quadriceps muscles (33) (circled in Fig. 2B). This electrically evoked protocol induced fatigue of the quadriceps independent of the central nervous system, although evoked contractions preferentially recruit and depolarize high threshold motor units that possess muscle fibers that are more easily fatigued. Women had a lower peak rate of relaxation indicating that their quadriceps muscles were slower than those of men. The initial peak rate of relaxation correlated with the reduction in torque. When the task was submaximal and involved the preferential recruitment of Type II fibers presumably for both men and women, the sexrelated difference in skeletal muscle fatigue resistance was not explained by a difference in muscle size (measured with magnetic resonance imaging, MRI) or strength (33). Contractile properties, however, indicated that the sex difference in muscle fatigue during isometric contractions could be attributed to a greater proportional area of Type II (fast twitch fibers) recruited during these contractions within the quadriceps muscles of the men compared with the women.

Similarly, contractile properties evoked with transcranial magnetic stimulation during maximal intermittent voluntary contractions of the elbow flexor muscles also illustrated the sex difference in muscle fatigue was associated with relaxation rates (12). Young men and women sustained six $22 \mathrm{~s}$ maximal isometric contractions. By the end of the six fatiguing MVCs, the men exhibited greater absolute and relative reductions in torque $(65 \pm 3 \%$ of initial MVC) than the women (52 $\pm 9 \%)$. The increments in torque (superimposed twitch) generated by motor cortex stimulation during each 22-s maximal effort increased with fatigue but did not differ between men and women, indicating fatigue upstream of the motor cortex (supraspinal fatigue) did not contribute to the sex difference in muscle fatigue during this task. The amplitude of the estimated resting twitch also decreased with fatigue during maximal isometric contractions; however, this reduction was greater for the men (59\%) than the women (27\%) (12). Parallel changes in the estimated resting twitch and maximal isometric contractions suggest that processes within the muscle largely explained the sex difference in muscle fatigue. The peak relaxation rates of the elbow flexors muscles (mainly biceps brachii) measured from twitches evoked with the cortical stimulation during the contractions were consistent with a greater proportional area of the muscle devoted to Type II (fast twitch) fibers in the men than the women. The men had faster relaxation rates prior to fatigue $\left(-13.5 \pm 2.2 \mathrm{~s}^{-1}\right)$ than women $\left(-9.3 \pm 1.8 \mathrm{~s}^{-1}\right)$ and the muscle slowed more during the fatigue task in the men (53\% from initial values) compared with the women (22 $\%$ from initial values). These findings indicated that the sex difference in muscle fatigue during maximal intermittent contraction with the elbow flexor muscles could be attributed to processes within the muscle.

Together, these studies with the knee extensor and elbow flexor muscles underscore the involvement of contractile mechanisms contributing to the sex differences in muscle fatigue during intermittent tasks in several large muscle groups. Comparison between findings for the dorsiflexor muscles $(28,29)$, elbow flexor muscles $(12)$ and knee extensor muscles 
$(23,33)$ indicate that the contribution of fiber type differences between men and women likely varies with the muscle involved.

\section{DYNAMIC CONTRACTIONS}

In contrast to isometric contractions, dynamic contractions do not show a systematic sex difference for most muscle groups. This is illustrated in Figure 1C for shortening contractions. There was no relation between contraction intensity and the sex difference for shortening contractions $\left(r^{2}=0.00005\right)$ when all muscle groups were pooled. How the sex difference in muscle fatigue can be altered with the contraction type was illustrated in a study that required 10 young men and 10 young women to perform a sustained isometric contraction and a fatiguing dynamic contraction until task failure with the back extensor muscles (4). Women had a longer time to task failure with the back extensor muscles than men for the isometric contraction sustained at $50 \%$ of maximal strength $(146 \pm 11 \mathrm{~s}$ vs $105 \pm$ $8 \mathrm{~s}$, respectively) (4). There was no sex difference, however, in the number of repeated shortening contractions performed at 50\% of maximal strength until the subject was not able to sustain the required velocity of contraction or range of motion $(24.3 \pm 3 \mathrm{vs} 24.0 \pm 3$ repetitions) (4). The reason for the absence of a sex difference in muscle fatigue during the dynamic contractions could be related to the requirement to maintain a given velocity during the shortening contractions. If women have a greater proportional area of Type I fibers, their muscles would possess a reduced maximal velocity of contraction compared with men. The velocity requirements of the task, therefore, may influence the ability to maintain a given load differently in men and women.

Close examination of Figure 1C indicates that a sex difference in muscle fatigue during shortening contractions can depend on the muscles involved. Figure $1 \mathrm{C}$ shows there is no sex difference in muscle fatigue for shortening contractions with leg and back muscles. The exception is the elbow flexor muscles, which show a clear sex difference in muscle fatigue at intermediate contraction intensities (50\% - 70\% of maximal strength) (25) and a negative association between the sex difference and contraction intensity $\left(\mathrm{r}^{2}=0.97\right)$ (Fig. 1C). The sex difference in performance during shortening contractions could be due to the large difference in the elbow flexor muscle mass between men and women relative to other muscles (25). Nevertheless, the few studies featured indicate more research is required to understand the role of the involved muscle group and the contraction velocity in determining any sex differences in muscle fatigue in functionally relevant dynamic contractions.

\section{MUSCLE GROUP}

There are few controlled studies that have assessed the sex difference in muscle fatigue across different muscles. The sex difference in performance of a fatiguing contraction, however, may not be as large for some muscles compared with others. For example, many studies show no sex difference for the dorsiflexor muscles for submaximal and maximal contractions $(10,22,29,30)$. In contrast, sex differences are found in most studies for the elbow flexor muscles at low- and high forces $(12,13,15,17,25,35)$. If the magnitude of the sex difference in muscle fatigue varies between muscle groups then the contributing mechanisms will differ. Of equal importance to understand, is that the magnitude of the sex difference in muscle fatigue may not change between muscles, yet the contributing mechanisms could vary.

The contribution of neural mechanisms to the sex difference in muscle fatigue can vary with the muscle group involved. One method to assess fatigue within the central nervous system involves stimulating the nervous system during maximal contractions to quantify the magnitude of voluntary drive to the muscle (voluntary activation) during or after a fatiguing contraction (9). Any observed increase in the increment in contraction force evoked by the 
superimposed stimulation implies a failure of voluntary drive at one or more sites proximal to the site of stimulation. A failure in voluntary activation during maximal efforts means that the level of neural drive to the muscle is less than optimal because either the motor units were not all recruited voluntarily, or they were discharging at rates that were not high enough to realize the force capacity of the muscle (9).

There was no sex difference in the reduction in voluntary activation during and after isometric fatiguing contractions performed with the elbows flexor muscles $(12,35)$. Voluntary activation assessed at the motor nerve was reduced similarly in young men and women (14\% reduction) after a low-force fatiguing contraction sustained with the elbow flexor muscles (35). Similarly, men and women exhibited similar levels of voluntary activation ( $\sim 77 \%$ for both sexes) at the end of six $22 \mathrm{~s}$ sustained maximal contractions as assessed with transcranial magnetic stimulation to quantify the magnitude of supraspinal fatigue (12). Thus, fatigue within the central nervous system was similar for men and women at the end of the low and high-force fatiguing contractions sustained with the elbow flexor muscles.

In contrast, the greater reduction in knee extensor force for men (24\%) compared with women $(16 \%)$ during a sustained maximal contraction was due to larger reductions in voluntary activation in men (22\%) than women (9\%) when assessed with evoked contractions elicited at the femoral nerve (23). Similarly, men had greater decrements in voluntary activation assessed with peroneal nerve stimulation during intermittent maximal contractions with the ankle dorsiflexor muscles (28). Thus, a sex difference in muscle fatigue was explained by greater reductions in voluntary activation for men compared with women for both the knee extensor muscles and the dorsiflexor muscles (Fig. 3).

The difference in contribution of neural mechanisms to the sex difference in muscle fatigue may be due to a difference in afferent feedback among muscle groups (Fig. 3). The firing of group III and IV muscle afferents, which are sensitive to ischemia and the metabolites of fatigue, can act at a supraspinal or spinal level to impair voluntary activation $(9,24)$. Higher intramuscular pressure in stronger muscles or sex differences in muscle metabolism and metabolic by-products $(6,29)$ can lead to greater discharge of group III and IV muscle afferents in men than women. Although group III and IV afferents do not inhibit motor neuron activity of the elbow flexor muscles (24), stimulation of the descending tracts shows that the motor neuron pool of the elbow extensors muscles is depressed by maintained firing of group III and IV muscle afferents after a fatiguing contraction (24). Thus, differences in the contribution of fatigue within the central nervous system to the sex difference in muscle fatigue maybe due to the sex-specific actions of group III and IV afferents onto the motor neuron pool in extensor and flexor muscles. This explanation is consistent among these muscle groups because the ankle dorsiflexor muscles are embryonically extensor muscles. Consequently, it is conceivable that the site within the central nervous system for the sex difference in muscle fatigue varies with the muscle being examined.

\section{COGNITIVE LOAD DURING A FATIGUING CONTRACTION}

The contribution of the neural mechanisms to the sex difference in performance of a fatiguing contraction for a given muscle group can increase by varying the task requirements. A role for supraspinal mechanisms in contributing to a sex difference in time to task failure has been demonstrated by increasing the cognitive load for men and women during a fatiguing contraction. A recent study showed that young women had greater reductions in the time to task failure of a low-force contraction than men when the gain of the visual feedback was altered so that the task required more attention (26). Fifteen young men and 14 young women maintained a constant elbow angle supporting a load at the wrist 
equivalent to $15 \%$ of maximal strength. The task was performed on separate days with either a low-gain or high-gain visual feedback of the joint angle signal ( 230 fold difference between conditions). The difference in visual feedback required greater attention during the high-gain task than the low-gain task despite a similar load torque being exerted. The men had a similar time to task failure for the low- and high-gain conditions $(6.0 \pm 2.2 \mathrm{~min}$ vs 5.9 $\pm 2.1 \mathrm{~min}$, respectively). In contrast, the women experienced a $27 \%$ reduction in the time to task failure for the high-gain task compared with the low-gain task $(8.7 \pm 2.3$ min vs $11.9 \pm$ 4.8 min respectively). Accordingly, sense of effort was greater for the women during the high-gain task.

Time to failure for the women was associated with the rate of decline in single motor unit discharge, changes in motor output, and increase in mean arterial pressure and heart rate during the fatiguing contraction. In contrast, the time to failure for the men was associated with the absolute target force, changes in motor output, and the EMG activity of the brachialis muscle. Despite each subject exerting the same net muscle torque during the two gain conditions, the time to failure for the fatiguing contractions was limited by different mechanisms for the men and women. This study also illustrated that increased attention required during the task altered the time to task failure for the women yet not for the men so that the sex difference in performance was diminished during the cognitively demanding task. The cause for the sex-specific impairment in performance may involve supraspinal mechanisms that mediate the stress response. Women have a greater physiological responses to stress-inducing stimuli than men $(2,21)$ and different brain activation patterns during a cognitive stressor compared with men (32).

To distinguish whether increased arousal (stress) and mental load contributed to the increased fatigability of the women compared with the men, young men and women performed a cognitive stressor while sustaining a fatiguing contraction with the elbow flexor muscles (34). Twenty young adults (10 men and 10 women) performed an isometric fatiguing contraction at $20 \%$ MVC force until task failure during two separate sessions. Each participant performed a mental-math task during one of the fatiguing contractions, which increased levels of anxiety and stress (stressor session): each subject was required to count backwards in increments of 13 or 7 from a four-digit number. Measures of arousal including salivary cortisol levels, and reported levels of anxiety were elevated during the stressor session compared with control. Maximal strength was similar at the start of the control and stressor sessions, and reduced similarly after the fatiguing contractions for both sexes. Time to task failure, however, was reduced for the women during the stressor session compared with the control session $(27.3 \pm 20.1 \%)$ but not for the men $(8.6 \pm 23.1 \%)$ (Fig.

$5 \mathrm{~A})$. The lesser time to task failure for the women was accompanied by higher mean arterial pressure and heart rates during the fatiguing contraction in the stressor session compared with the control task (Fig. 5B-C).

The longer time to failure for the women observed under control conditions disappeared with the stressful dual task and the physiological responses (particularly indicators of sympathetic nerve activity) of the women were more similar to the men. Despite a similar force requirement during the two tasks, the increased cognitive load and anxiety resulted in altered physiological adjustments including indices of sympathetic control during the fatiguing contraction that were associated with greater fatigability in the women but not the men. The magnitude of reduction in the time to task failure of the fatiguing contraction for the women was similar when imposing a stressful cognitive task (34) and when visual task feedback was at a higher gain (26) (27\% difference for both studies). Taken together, the above studies highlight the important role of supraspinal centers, and cognitive demand and arousal on performance during low-force fatiguing contractions performed by men and women. 


\section{AGE AND SEX DIFFERENCES}

Lastly, the age of the individual will influence the magnitude of the sex difference in time to failure of a submaximal task. All the examples discussed so far in this review and the results of studies plotted in Figure 1 involve young adults only. The collective studies shown in Figure 2, however, demonstrate that the magnitude of the sex difference in performance of a fatiguing contraction is lessened or disappears among older adults. The plotted linear regression of dynamic and isometric tasks $\left(r^{2}=0.22\right)$ indicates that the sex difference is negligible at 72 years of age. Separate analysis of the isometric and dynamic tasks, however, indicate that only isometric contractions showed a significant negative relation $\left(\mathrm{r}^{2}=0.30\right)$ between the sex difference and age. Tasks involving shortening contractions showed no change across age groups $\left(r^{2}=0.02\right)$ because the sex difference was already small for dynamic tasks in young adults (Fig. 1C).

These findings for an isometric contraction with age were clarified in a study that required 27 young adults ( 14 men, 13 women, $18-35$ years) and 18 old (10 men, 8 women, 65-80 years) adults to sustain an isometric contraction at $20 \%$ of MVC torque until task failure (13). The old women were not on hormone replacement therapy and had been postmenopausal for $>10$ years. The young adults $(67 \pm 18 \mathrm{~N} \bullet \mathrm{m})$ were stronger than the old adults $(48 \pm 18 \mathrm{~N} \bullet \mathrm{m})$ and the men $(70 \pm 18 \mathrm{~N} \bullet \mathrm{m})$ were stronger than the women $(47 \pm 15$ $\mathrm{N} \bullet \mathrm{m})$. The time to task failure was longer for the young women $(18.3 \pm 8.0 \mathrm{~min})$ compared with the young men $(10.8 \pm 5.2 \mathrm{~min})$, but there was no sex difference between the old women $(21.3 \pm 10.7 \mathrm{~min})$ and $\mathrm{men}(24.1 \pm 8.0 \mathrm{~min})$ or between the young women and old adults (Fig. 6). The time to task failure was related to the absolute target torque exerted for the young adults as shown for this task previously (17), but not for the old adults. The differences in task duration among subject groups were accompanied by parallel changes in the increase in mean arterial pressure (pressor response) (13). Because there was no association between absolute target force and time to failure in the old adults, some other mechanism that did not involve contraction strength contributed to the reduced pressor response and similar times to failure of the older men and women. This study demonstrates that the mechanisms for the sex difference in performance of a fatiguing contraction change with increased age and are specific to the men.

The reduction in the sex difference in time to failure with age raises the question of whether hormonal differences between men and women mediate the sex difference in muscle fatigue and performance of fatiguing contractions among young adults. Because the time to failure was similar in the young and old women (13), age-related changes in estrogen and progesterone are not implicated, despite their possible influence on sympathetic activity, the pressor response, and muscle metabolism during the menstrual cycle in young women (7). Accordingly, there is little evidence from other studies to suggest that performance of a fatiguing contraction differs across the menstrual cycle and the associated fluctuations in hormonal level in young women $(14,15,20)$. Furthermore, there was no difference in time to failure of an intermittent task performed with the adductor pollicis muscle at 50\% MVC among older women who were on hormone replacement therapy and those who were not (1). The briefer time to task failure of the young men compared with the young women and old men and women (16), therefore, is likely related to some mechanisms specific to the young men.

\section{CONCLUSIONS AND FUTURE DIRECTIONS}

Women can be less fatigable than men for many muscle groups under controlled conditions during isometric contractions. When the task demands are altered, for example by increasing contraction intensity, increasing the cognitive load during a contraction, changing the 
contraction type to involve dynamic contractions, or assessing old adults, the magnitude of the sex difference is diminished. Accordingly, the magnitude of a sex-difference in muscle fatigue is specific to the task performed and other task details including the involved muscle group and the age of the individual assessed. This task specificity for the magnitude of the sex difference in muscle fatigue is due to sex-related differences within the neuromuscular system. The rate-limiting mechanism(s) that constrains performance of a fatiguing contraction differs between men and women. Thus, the strength of a contributing mechanism to the sex difference in performance during a fatiguing contraction will alter as the task is varied because different tasks stress different physiological systems.

Consequently, there is not one global cause for the sex difference in muscle fatigue.

Low-force fatiguing contractions are the foundation of many daily tasks and are also a precursor to work-related disorders and pain. In contrast, muscle fatigue is necessary for effective neuromuscular adaptation during training and rehabilitation. The challenge for future studies is to identify those tasks that limit performance of functionally relevant fatiguing tasks in men and women differently. An even greater challenge is to understand the rate-limiting physiological adjustments that cause the sex difference for a specific task. In doing so, sex-specific strategies can be identified for effective rehabilitation or alternatively to offset fatigue during functionally relevant daily tasks.

\section{Acknowledgments}

The author thanks Jeffrey Rainwater for his contribution to Figure 3 and Carol Mottram, Ph.D. and Marie Hoeger Bement, Ph.D. for their comments on the manuscript.

Funding: Funding was provided by NIH/NIOSH and Marquette University.

\section{REFERENCES}

1. Cheng A, Ditor DS, Hicks AL. A comparison of adductor pollicis fatigue in older men and women. Can J Physiol Pharmacol 2003;81:873-879. [PubMed: 14614523]

2. Christou EA, Jakobi JM, Critchlow A, Fleshner M, Enoka RM. The 1- to 2-Hz oscillations in muscle force are exacerbated by stress, especially in older adults. J Appl Physiol 2004;97:225-235. [PubMed: 15220319]

3. Clark BC, Collier SR, Manini TM, Ploutz-Snyder LL. Sex differences in muscle fatigability and activation patterns of the human quadriceps femoris. Eur J Appl Physiol 2005;94:196-206. [PubMed: 15791418]

4. Clark BC, Manini TM, The DJ, Doldo NA, Ploutz-Snyder LL. Gender differences in skeletal muscle fatigability are related to contraction type and EMG spectral compression. J Appl Physiol 2003;94:2263-2272. [PubMed: 12576411]

5. Enoka RM, Duchateau J. Muscle fatigue: what, why and how it influences muscle function. J Physiol 2008;586:11-23. [PubMed: 17702815]

6. Ettinger SM, Silber DH, Collins BG, Gray KS, Sutliff G, Whisler SK, McClain JM, Smith MB, Yang QX, Sinoway LI. Influences of gender on sympathetic nerve responses to static exercise. J Appl Physiol 1996;80:245-251. [PubMed: 8847310]

7. Ettinger SM, Silber DH, Gray KS, Smith MB, Yang QX, Kunselman AR, Sinoway LI. Effects of the ovarian cycle on sympathetic neural outflow during static exercise. J Appl Physiol 1998;85:2075-2081. [PubMed: 9843528]

8. Fulco CS, Rock PB, Muza SR, Lammi E, Cymerman A, Butterfield G, Moore LG, Braun B, Lewis SF. Slower fatigue and faster recovery of the adductor pollicis muscle in women matched for strength with men. Acta Physiol Scand 1999;167:233-239. [PubMed: 10606825]

9. Gandevia SC. Spinal and supraspinal factors in human muscle fatigue. Physiol Rev 2001;81:17251789. [PubMed: 11581501] 
10. Griffith EE, Yoon T, Farinella J, Ng AV, Hunter SK. Type of feedback and time to task failure of young and old adults. Med Sci Sports Exerc 2008;40:S448.

11. Hicks AL, Kent-Braun J, Ditor DS. Sex differences in human skeletal muscle fatigue. Exerc Sport Sci Rev 2001;29:109-112. [PubMed: 11474957]

12. Hunter SK, Butler JE, Todd G, Gandevia SC, Taylor JL. Supraspinal fatigue does not explain the sex difference in muscle fatigue of maximal contractions. J Appl Physiol 2006;101:1036-1044. [PubMed: 16728525]

13. Hunter SK, Critchlow A, Enoka RM. Influence of aging on sex differences in muscle fatigability. J Appl Physiol 2004;97:1723-1732. [PubMed: 15208285]

14. Hunter SK, Critchlow A, Shin IS, Enoka RM. Fatigability of the elbow flexor muscles for a sustained submaximal contraction is similar in men and women matched for strength. J Appl Physiol 2004;96:195-202. [PubMed: 14514707]

15. Hunter SK, Critchlow A, Shin IS, Enoka RM. Men are more fatigable than strength-matched women when performing intermittent submaximal contractions. J Appl Physiol 2004;96:21252132. [PubMed: 14966025]

16. Hunter SK, Duchateau J, Enoka RM. Muscle fatigue and the mechanisms of task failure. Exerc Sport Sci Rev 2004;32:44-49. [PubMed: 15064647]

17. Hunter SK, Enoka RM. Sex differences in the fatigability of arm muscles depends on absolute force during isometric contractions. J Appl Physiol 2001;91:2686-2694. [PubMed: 11717235]

18. Hunter SK, Griffith EE, Schlachter KM, Kufahl TD. Sex differences in time to task failure and blood flow for an intermittent isometric fatiguing contraction. Muscle Nerve 2009;39:42-53. [PubMed: 19086076]

19. Hunter SK, Schletty JM, Schlachter KM, Griffith EE, Polichnowski AJ, Ng AV. Active hyperemia and vascular conductance differ between men and women for an isometric fatiguing contraction. $\mathrm{J}$ Appl Physiol 2006;101:140-150. [PubMed: 16601303]

20. Janse de Jonge XA. Effects of the menstrual cycle on exercise performance. Sports Med 2003;33:833-851. [PubMed: 12959622]

21. Kajantie E, Phillips DI. The effects of sex and hormonal status on the physiological response to acute psychosocial stress. Psychoneuroendocrinology 2006;31:151-178. [PubMed: 16139959]

22. Kent-Braun JA, Ng AV, Doyle JW, Towse TF. Human skeletal muscle responses vary with age and gender during fatigue due to incremental isometric exercise. J Appl Physiol 2002;93:18131823. [PubMed: 12381770]

23. Martin PG, Rattey J. Central fatigue explains sex differences in muscle fatigue and contralateral cross-over effects of maximal contractions. Pflugers Arch 2007;454:957-969. [PubMed: 17342531]

24. Martin PG, Smith JL, Butler JE, Gandevia SC, Taylor JL. Fatigue-sensitive afferents inhibit extensor but not flexor motoneurons in humans. J Neurosci 2006;26:4796-4802. [PubMed: 16672652]

25. Miller A, MacDougall J, Tarnopolsky M, Sale D. Gender differences in strength and muscle fiber characteristics. Eur J Appl Physiol 1993;66:254-262.

26. Mottram CJ, Hunter SK, Rochette L, Anderson MK, Enoka RM. Time to task failure varies with the gain of the feedback signal for women, but not for men. Exp Brain Res 2006;174:575-587. [PubMed: 16680423]

27. Roepstorff C, Thiele M, Hillig T, Pilegaard H, Richter EA, Wojtaszewski JF, Kiens B. Higher skeletal muscle alpha2AMPK activation and lower energy charge and fat oxidation in men than in women during submaximal exercise. J Physiol 2006;574:125-138. [PubMed: 16600998]

28. Russ DW, Kent-Braun JA. Sex differences in human skeletal muscle fatigue are eliminated under ischemic conditions. J Appl Physiol 2003;94:2414-2422. [PubMed: 12562681]

29. Russ DW, Lanza IR, Rothman D, Kent-Braun JA. Sex differences in glycolysis during brief, intense isometric contractions. Muscle Nerve 2005;32:647-655. [PubMed: 16025523]

30. Russ DW, Towse TF, Wigmore DM, Lanza IR, Kent-Braun JA. Contrasting influences of age and sex on muscle fatigue. Med Sci Sports Exerc 2008;40:234-241. [PubMed: 18202580] 
31. Thompson BC, Fadia T, Pincivero DM, Scheuermann BW. Forearm blood flow responses to fatiguing isometric contractions in women and men. Am J Physiol Heart Circ Physiol 2007;293:H805-H812. [PubMed: 17468333]

32. Wang J, Korczykowski M, Rao H, Fan Y, Pluta J, Gur RC, McEwen BS, Detre JA. Gender difference in neural response to psychological stress. Soc Cogn Affect Neurosci 2007;2:227-239. [PubMed: 17873968]

33. Wust RC, Morse CI, de Haan A, Jones DA, Degens H. Sex differences in contractile properties and fatigue resistance of human skeletal muscle. Exp Physiol 2008;93:843-850. [PubMed: 18296492]

34. Yoon, T.; Schlinder-Delap, B.; Hunter, SK. Program No 6504/X3 2006 Neuroscience Meeting Planner. Atlanta, GA: Society for Neuroscience; 2006. Performance of a cognitive task impairs time to failure and steadiness for a submaximal fatiguing contraction. Online: http://www.sfn.org

35. Yoon T, Schlinder Delap B, Griffith EE, Hunter SK. Mechanisms of fatigue differ after low- and high-force fatiguing contractions in men and women. Muscle Nerve 2007;36:512-524. 

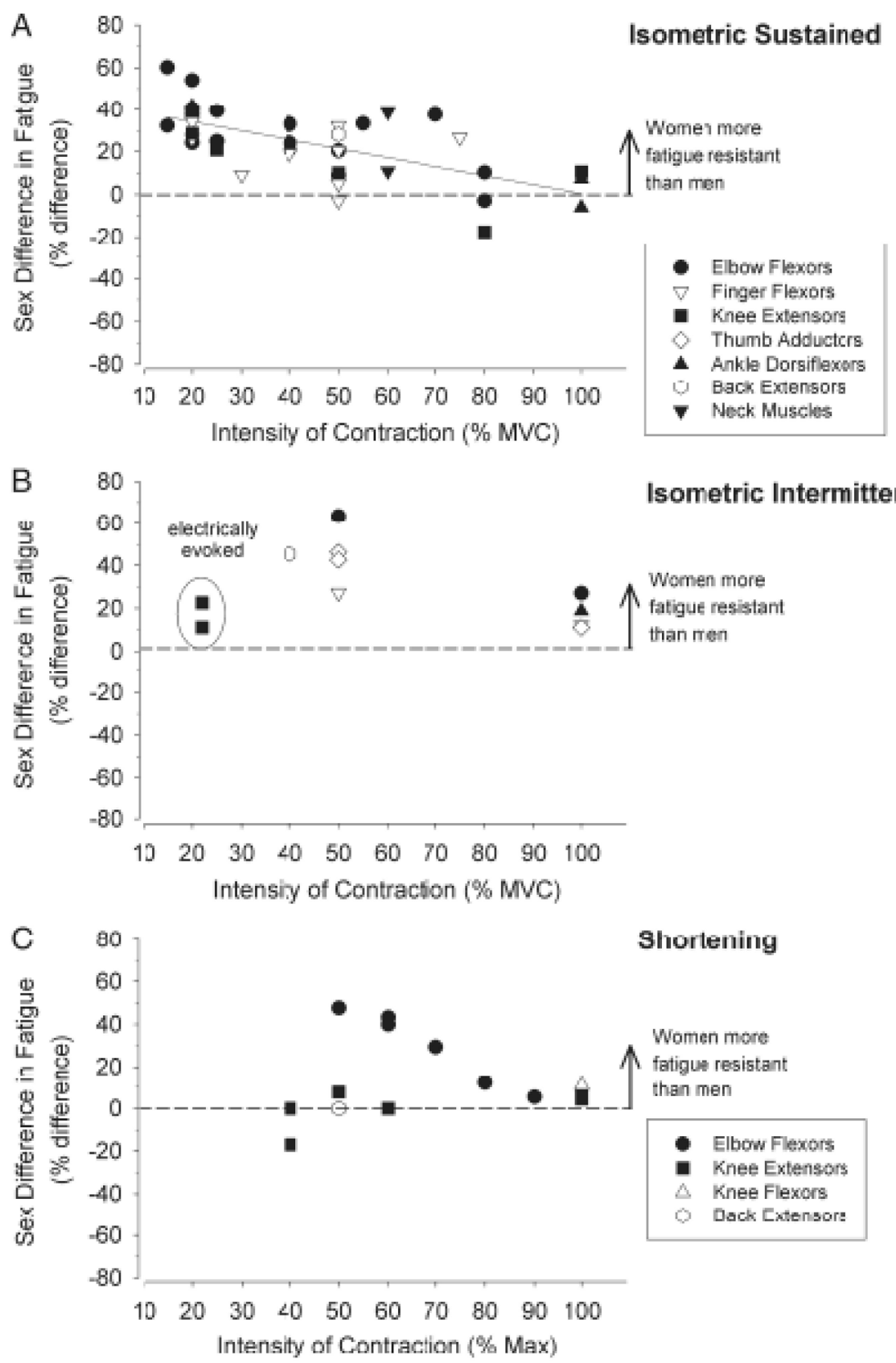

Figure 1. Sex differences in performance of a fatiguing contraction for isometric sustained contractions $(A)$, isometric intermittent contractions $(B)$ and shortening contractions $(C)$ Represented are mean data from 42 studies that assessed fatiguing contractions in men and women for various muscle groups. Plotted is the difference in the mean fatigue index or time to task failure (relative to the women) within a study between the young men and young women as a function of the contraction intensity. There was a significant negative relation between the relative contraction intensity and the magnitude of the sex difference for the isometric sustained contractions $\left(A ; \mathrm{r}^{2}=0.42\right)$ and the isometric intermittent contractions $\left(B ; \mathrm{r}^{2}=0.67\right)$. There was no relation between contraction intensity and the sex difference for shortening contractions $\left(C ; \mathrm{r}^{2}=0.00005\right)$ for all muscle groups pooled. Data from two 
studies for the elbow flexor muscles however, showed a significant negative relation $\left(\mathrm{r}^{2}=\right.$ 0.97 ) for shortening contractions $(C)$. There are more data points than number of stated studies because some studies involved multiple contraction types or intensities. 


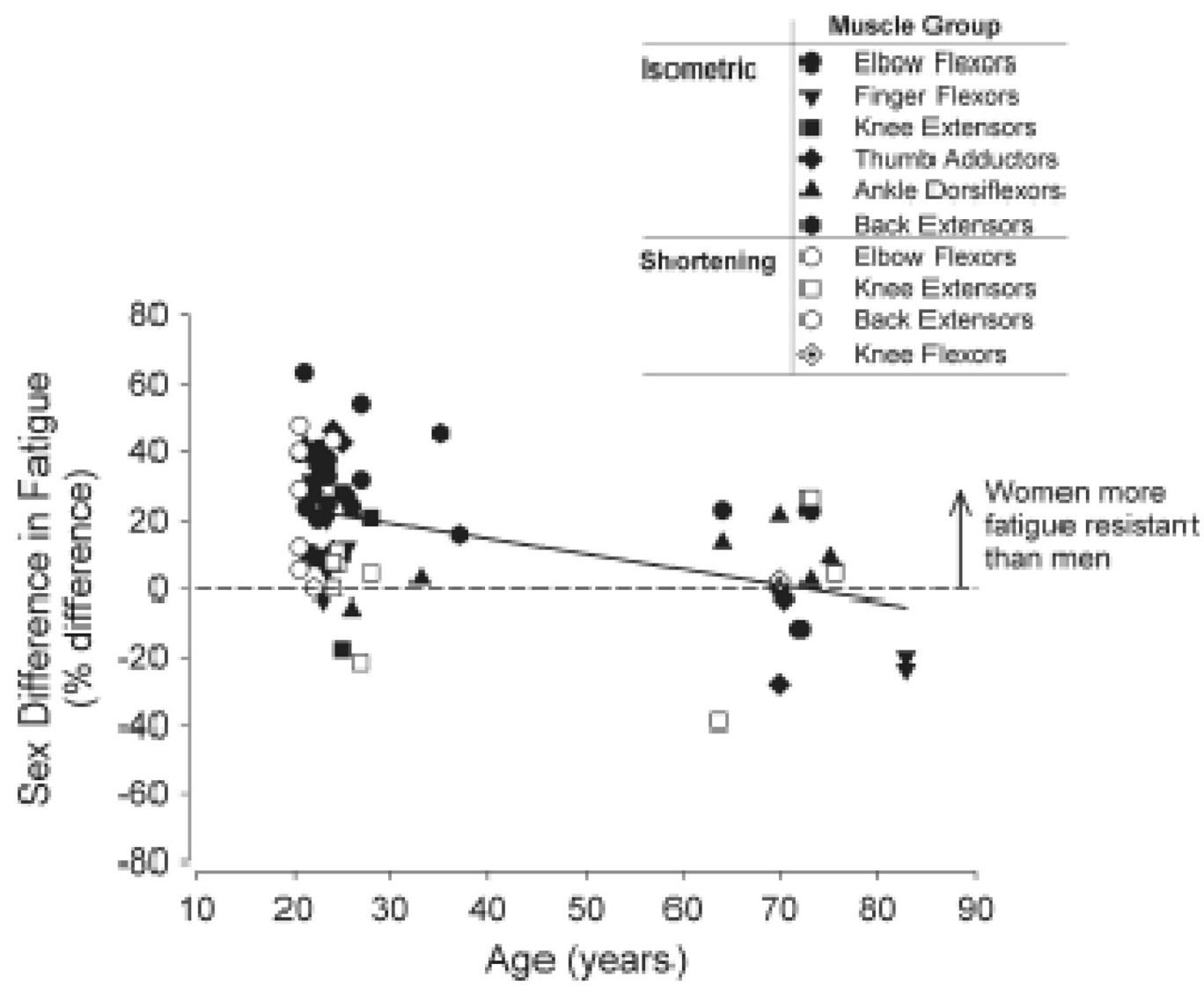

Figure 2. Sex differences in performance of a fatiguing contraction among young and old adults for isometric contractions (filled symbols), and dynamic contractions (open symbols)

Represented are mean data from 40 studies for various muscle groups. Plotted is the difference in the mean fatigue index or time to task failure (relative to the women) within a study between the men and women as a function of the mean age of the subject group assessed within the study. Sustained and intermittent isometric tasks are plotted as one group under isometric contractions. Linear regression for all the plotted data $\left(r^{2}=0.22\right)$ indicates that the sex difference is negligible at 72 years of age (regression line shown). The relation was significant for the isometric tasks only $\left(\mathrm{r}^{2}=0.30\right)$ and not the dynamic tasks involving shortening contractions $\left(r^{2}=0.02\right)$. 


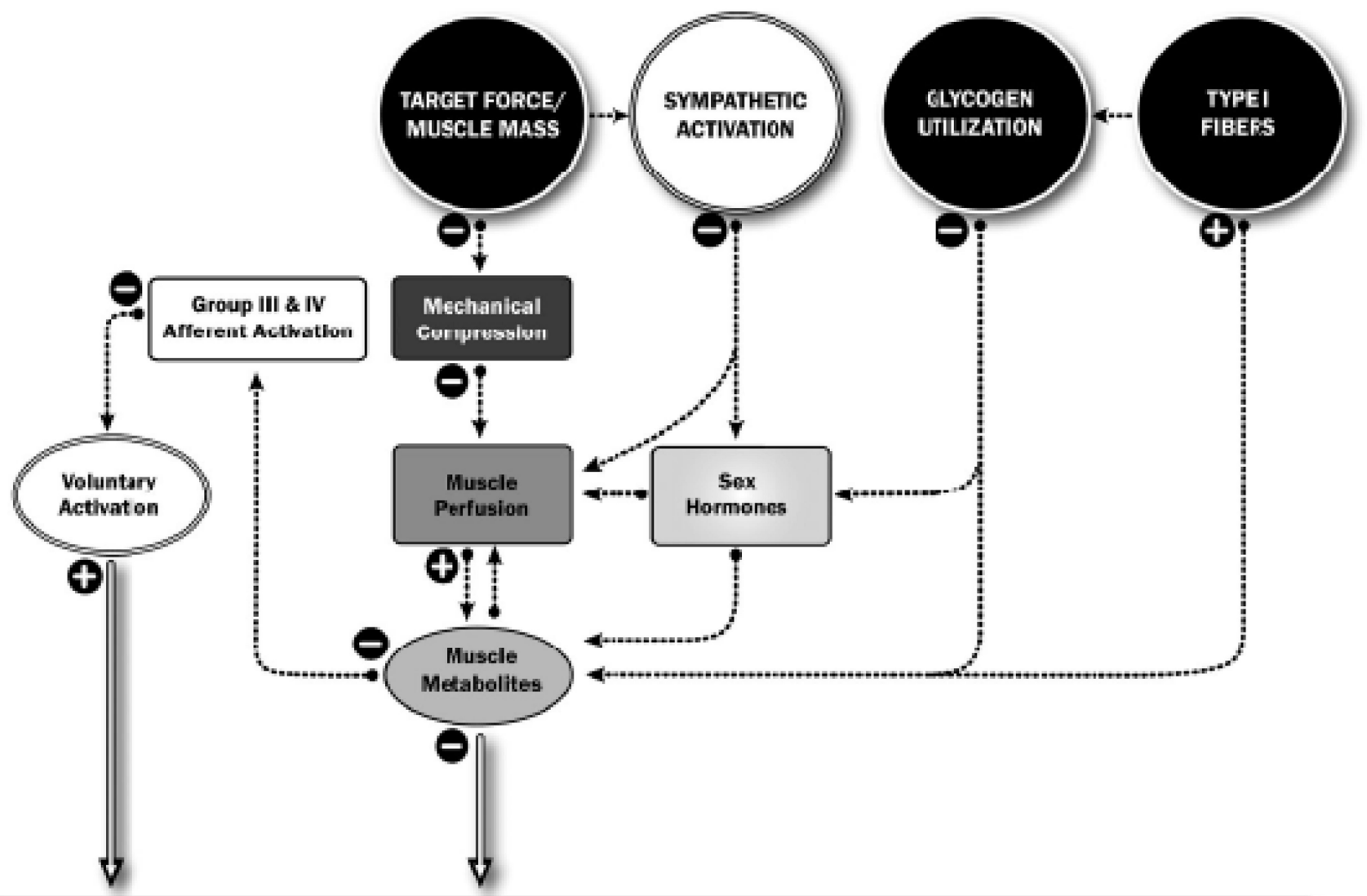

\section{WOMEN : Less Fatigable}

Less in women than in men

Greater in women than in men

Figure 3. Potential mechanisms for the sex difference in performance of a fatiguing contraction (muscle fatigue or time to task failure)

The figure shows those potential mechanisms that can cause women to be more fatigue resistant than men. Neural mechanisms are in white and muscular mechanisms are shaded. The strength of a potential mechanism will vary with the task conditions so that one dominant mechanism does not fully explain the sex difference in performance of a fatiguing contraction. A negative sign indicates that the physiological variable or process is less in women than men and conversely a positive sign indicates it is greater in women than men. This figure does not indicate the strength of the mechanism and is not specific to any one task. Only those variables that maybe to be implicated in women being less fatigable than men are shown. 

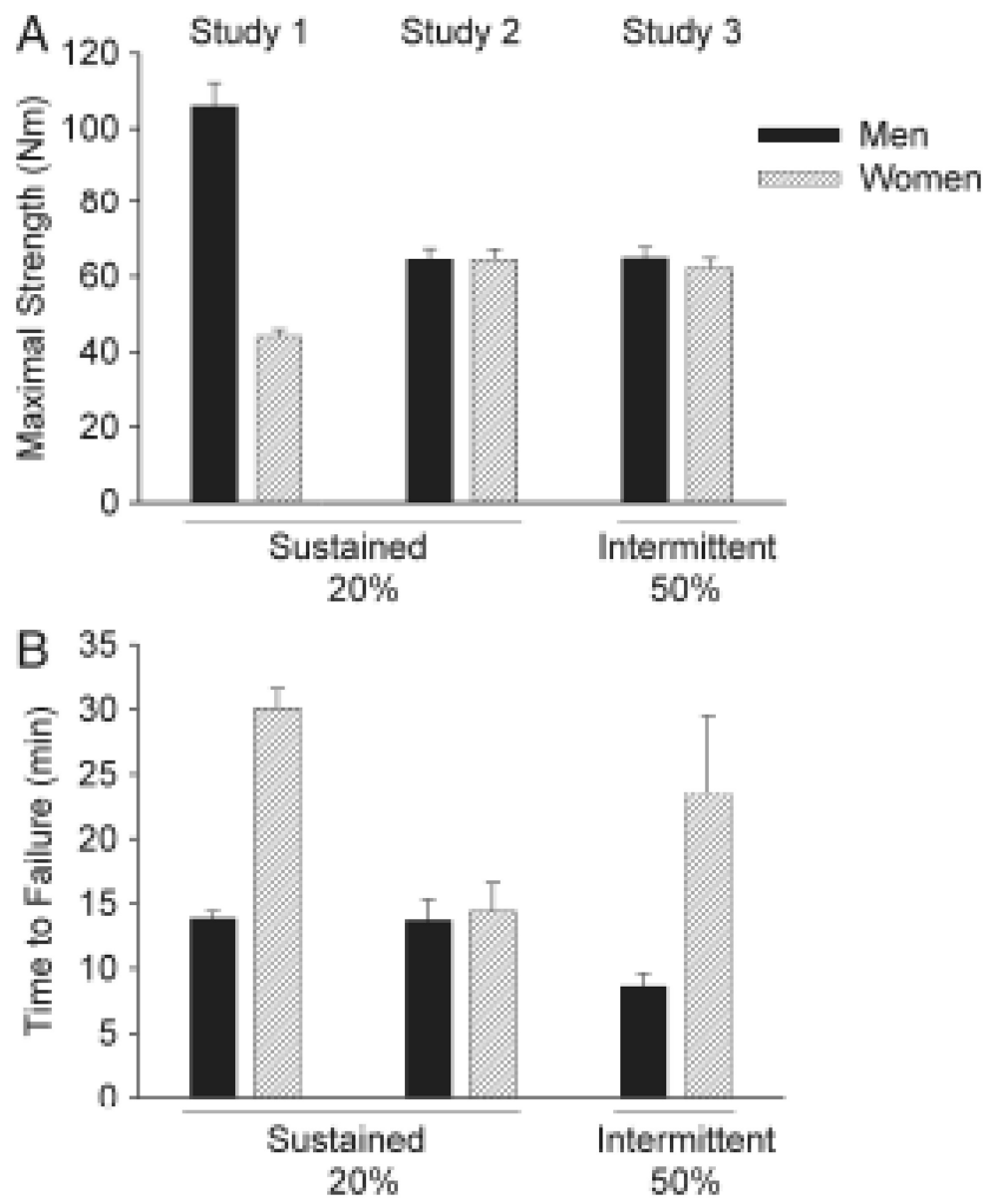

Figure 4. Maximal strength $(A)$ and time to task failure $(B)$ for isometric tasks sustained with the elbow flexor muscles in three separate studies

All data shown represent the mean \pm SEM values. A. Shown is maximal voluntary isometric strength performed prior to the fatiguing contraction by young men and young women for study 1 when men were stronger than women $(n=14)(17)$, and study $2(n=20)(14)$ and study $3(\mathrm{n}=20)(15)$ when men and women were matched for strength. $B$. The time to task failure for each of the tasks performed in the three studies are shown. Studies 1 and 2 involved a sustained isometric task at 20\% MVC. Study 3 involved an intermittent isometric task performed at $50 \%$ of MVC. 

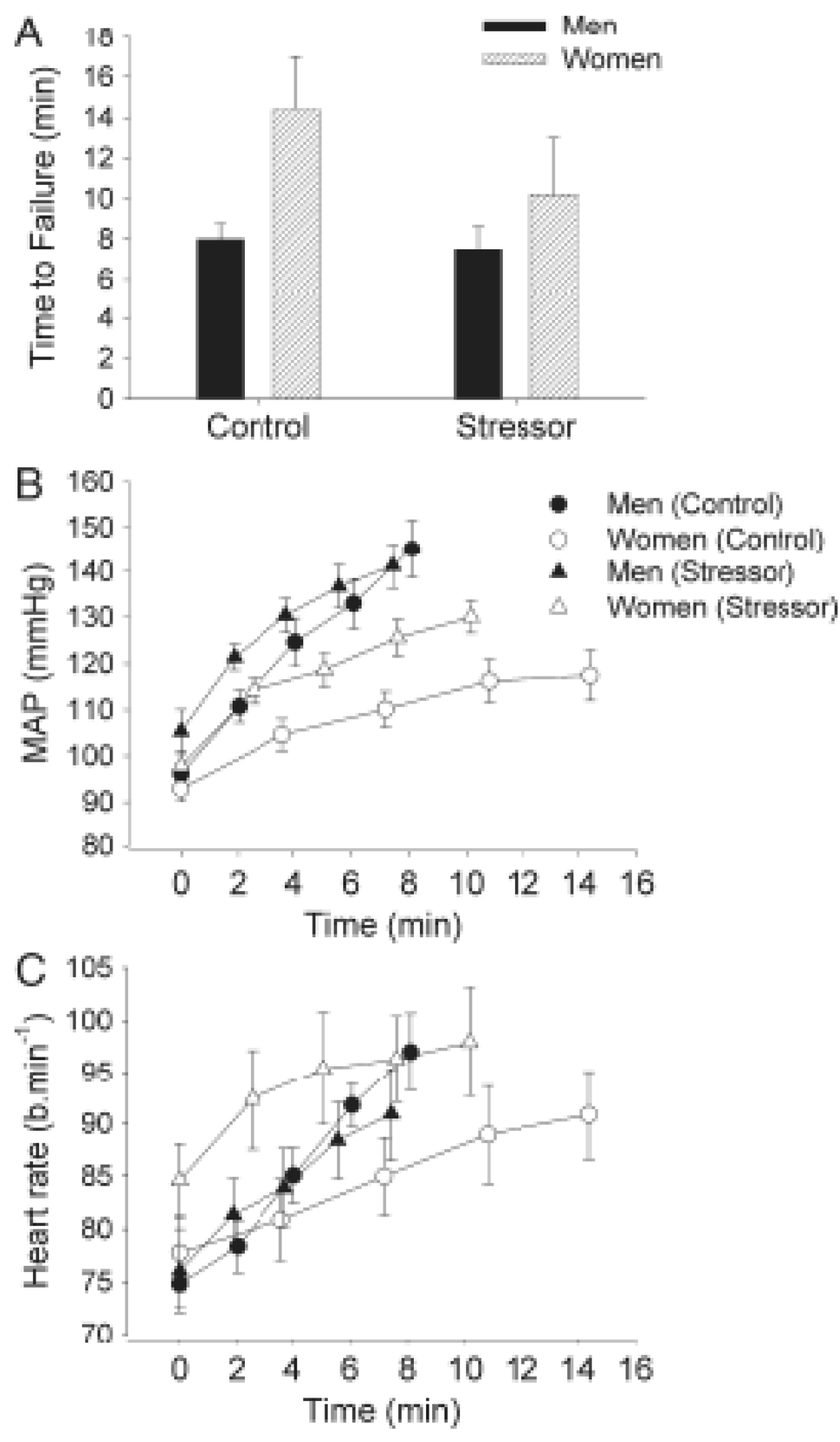

Figure 5. Sex differences in performance and cardiovascular control for a fatiguing contraction with elbow flexor muscles when a stressful cognitive load is imposed

$A$. Time to task failure (mean \pm SEM) for an isometric fatiguing contraction sustained at $20 \%$ of maximal strength by young men and women during a stressor session and control session. The stressor session required the subject to perform a difficult mental- math task while performing the fatiguing contraction. There was a sex by session interaction $(P<0.05)$ because the women had a reduction in time to failure during the stressor session but the men did not. $B-C$. Mean arterial pressure (MAP; $B)$ and heart rate $(C)$ during the fatiguing contraction. The values are the mean $\pm \mathrm{SE}$ at $25 \%$ increments of the time to task failure for men (closed symbols) and women (open symbols) during the control session (circles) and stressor session (triangles). Averages of 15-s intervals were used for the MAP and heart rate. 
MAP and heart rate were higher during the stressor task, especially for the women. Data from Yoon et al. (2006) (34). 


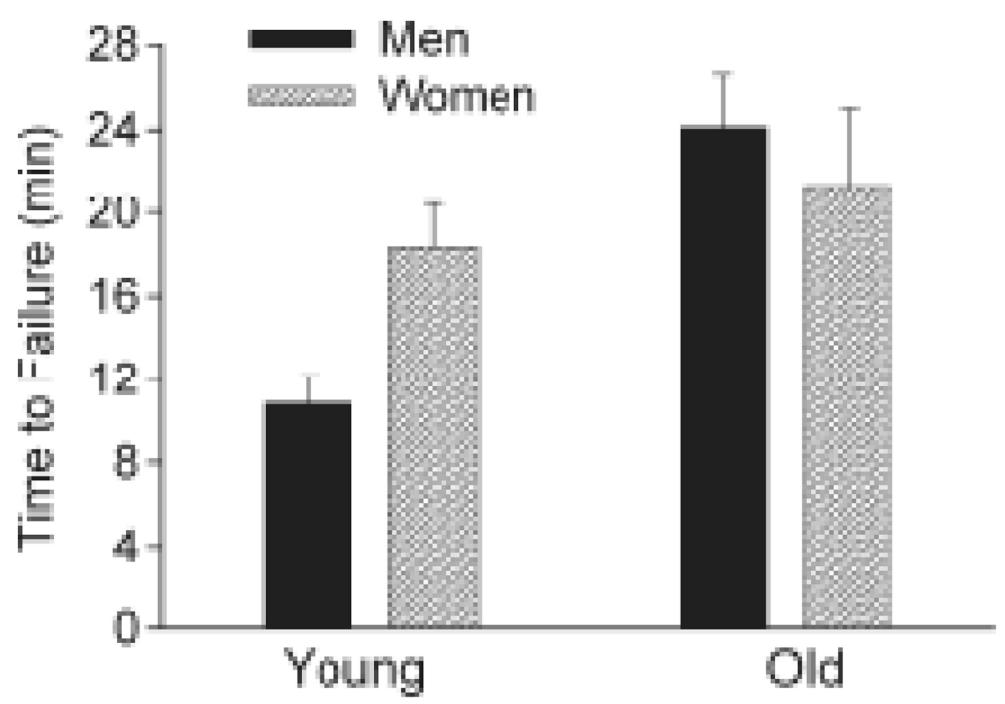

Figure 6.

Time to task failure for a sustained isometric fatiguing contraction performed with elbow flexor muscles at $20 \%$ of maximal strength by young men $(n=14)$, young women $(n=13)$, old men $(n=10)$ and old women $(n=8)$. Time to task failure (mean \pm SEM) was longer for the young women compared with the young men but similar for old men and women. [Adapted from Hunter SK, Critchlow A, Enoka RM. Influence of aging on sex differences in muscle fatigability. J Appl Physiol. 2004;97:1723-32. Copyright (C) 2004 The American Physiological Society. Used with permission.] 